\title{
Outcome estimation based on multimodal computed tomography examination in acute ischaemic stroke patients treated with mechanical thrombectomy
}

\author{
Bartłomiej Łasocha1 ${ }^{1}$, Paweł Brzegowy ${ }^{2}$, Agnieszka Słowik ${ }^{3}$, Paweł Latacz ${ }^{3}$, Roman Pułyk ${ }^{3}$, Tadeusz J. Popiela² \\ ${ }^{1}$ Department of Rescue Medicine and Multiorgan Truma, Diagnostic Imaging Unit, University Hospital, Krakow, Poland \\ ${ }^{2}$ Chair of Radiology, Jagiellonian University Medical College, Krakow, Poland \\ ${ }^{3}$ Chair of Neurology, Jagiellonian University Medical College, Krakow, Poland
}

Videosurgery Miniinv 2019; 14 (4): 560-566

DOI: https://doi.org/10.5114/wiitm.2019.84761

\begin{abstract}
Introduction: Reperfusion therapy for acute ischaemic stroke used within a time window of $6 \mathrm{~h}$ following symptom onset, although currently the treatment of choice, is characterised by certain limitations and carries certain risks.

Aim: To assess the potential for predicting the risks and limitations of reperfusion therapy by means of advanced neuroimaging.

Material and methods: For this purpose, the baseline CT scans of patients with ischaemic stroke treated by means of mechanical thrombectomy were assessed retrospectively using the Combined Multimodal Computed Tomography Score (CMCTS), modified to account for the perfusion examination of a limited area. These data were then combined with radiological and clinical outcomes, in particular haemorrhagic stroke transformation and scoring on a modified Rankin scale (mRS).

Results: Based on material from 85 patients, the scoring system we employed enabled us to distinguish a group that did not benefit from treatment with specificity and a positive predictive value of $100 \%$, and a negative predictive value of $64 \%$. Neither the relationship between the score and early haemorrhagic complications, nor the effectiveness or severity of the course of the thrombectomy procedure itself was confirmed.

Conclusions: There is no justification for the use of reperfusion procedures in acute ischaemic stroke in the anterior circulation in patients with initially unfavourable multimodal computed tomography scores.
\end{abstract}

Key words: stroke, computed tomography, mechanical thrombectomy.

\section{Introduction}

Over the past decade, we have observed the rapid development of methods of causal treatment of acute cerebral ischaemia. One inseparable element of this development consists of changes in ways of thinking about the organisation of stroke treatment, patient qualification, prognostic factors, and mechanisms of the course of the disease - both natural and those with which medical treatment interferes
[1]. The milestones of mechanical thrombectomy, while leaving no doubt that this method is effective and necessary [2-5], have come at the cost of the exclusion of patients through the use of strict qualification criteria [6]. Measures commonly used in the diagnosis of acute stroke do not permit precise definition of the borderline beyond which patients will no longer benefit from interventional treatment [7]. The Combined Multimodal Computed Tomography

\section{Address for correspondence}

Bartłomiej Łasocha, Department of Rescue Medicine and Multiorgan Truma, Diagnostic Imaging Unit, University Hospital,

63 Kopernika St, 31-501 Krakow, Poland, phone: +48 1242477 74, e-mail: blasocha@su.krakow.pl 
Score (CMCTS) was introduced by de Rueda et al. in 2015 and verified through correlation with long term treatment results in a group of effective recanalisation [8]. The main difference between the score used in our study and the original version is our simplified evaluation of cerebral blood volume maps. Hereafter we will refer to our own results using the term Simplified Multimodal Computed Tomography Score (SMCTS).

\section{Aim}

The aim of the study was to evaluate the usefulness of SMCTS in acute ischaemic stroke. In developing the topic of safety and effectiveness of acute stroke treatment, we analysed the relationship of this score to the failure of recanalisation, procedural burden, and haemorrhagic complications.

\section{Material and methods}

The CMCTS was calculated from the sum of points awarded for three factors: poor collateral circulation, an unfavourable blood volume (BV) CT perfusion map, and extensive areas that failed to demonstrate contrast enhancement in initial CT angiography signal intensity (CTA-SI) images.

For the purpose of calculating the score, collateral circulation was evaluated as either good (0 points) or poor (1 point), depending on whether or not the vessels of the Sylvian fissure and hemisphere convexity were opacified in CT angiography.

The remaining parameters were evaluated similarly to plain CT images in the ASPECTS scale: in each of 10 areas isolated in the territory of the middle cerebral artery, corresponding features of ischaemia were assessed separately. Starting with a value of 10 , one point was subtracted for each area in which the relevant changes were observed. The results thus obtained, defined as BV ASPECTS and CTA-SI ASPECTS, were expressed in values within a range of 0-10. For the purposes of CMCTS, threshold values were adopted for both scores: a point on the scale was awarded for CTA-SI ASPECTS below 6, and for BV-ASPECTS less than 7. Poor collateral circulation also resulted in adding 1 to the CMCTS; hence this scale took values within the range $0-3$.

In our study, however, due to the narrow area imaged in the perfusion examination according to our protocol, BV ASPECTS scores were determined only partially. Because the area of the basal ganglia was always included, analysis of lenticular nuclei, internal capsules, caudal nuclei, insular cortex, and the M1-M3 areas of the ASPECT scale was carried out. The M4-M6 areas were included in the examined field only in some cases and therefore were not evaluated; moreover, no attempt was made to extrapolate their status based on the angioCT or plain CT findings. To assure compliance with the score definition and potential comparisons of measurements in other studies, it was assumed that they were intact and each scored as one point, yielding results for BV ASPECTS within the range 3-10.

Starting on 2 January 2013, of all patients admitted to hospital with a diagnosis of ischaemic stroke, 135 patients with large vessel occlusion were qualified for mechanical thrombectomy. Patients presenting within a time window of $6 \mathrm{~h}$ from the onset of symptoms for whom intravenous thrombolysis proved to be contraindicated or ineffective, as well as those diagnosed too late for application of intravenous fibrinolysis, were given endovascular treatment. Both the co-ordinating neurologist and the staff of the interventional neuroradiology suite were on call; the preliminary clinical and radiological assessment was made by neurologists and a radiologist on stationary duty.

Patients diagnosed in a local emergency department or during hospitalisation in local hospital wards were subjected to multimodal $\mathrm{CT}$, consisting of basic CT without contrast medium, CT perfusion, and CT angiography of extra- and intracranial arteries. Only middle cerebral artery strokes were included in the study.

All CT examinations were performed on a 64-slice OPTIMA CT660 scanner (GE Healthcare, Chicago, Illinois, USA). Non-enhanced brain CT scans were acquired via a sequential technique, reconstructing 8 layers, each $2.5 \mathrm{~mm}$ thick, per revolution. The remaining CT parameters were: $120 \mathrm{kV}$ potential, 150-180 mA current, $2 \mathrm{~cm}$ detector coverage, $0.625 \mathrm{~mm}$ single-layer collimation, 1.07 detector pitch, and $2 \mathrm{~s}$ rotation time. The CT perfusion was performed following administration of $45 \mathrm{ml}$ of contrast agent at the rate of $4 \mathrm{ml} / \mathrm{s}$, in a sequential algorithm, in a section $4 \mathrm{~cm}$ wide, divided into 8 layers, each $5 \mathrm{~mm}$ thick. The rotation time was $1 \mathrm{~s}$, the voltage of the lamp $80 \mathrm{kV}$, the anode current $120 \mathrm{~mA}$; each scan lasted $45 \mathrm{~s}$.

The vascular scan was acquired in a transverse plane, using a helical algorithm; the beginning of the 
acquisition was triggered using the bolus-tracking technique (SmartPrep) following the administration of $80 \mathrm{ml}$ of contrast medium at the rate of $4 \mathrm{ml} / \mathrm{s}$, followed by a $30-\mathrm{ml}$ saline bolus. The scan covered the area from the top of the head to the aortic arch. The lamp current was $320 \mathrm{~mA}$, its potential $120 \mathrm{kV}$; rotation time was reduced to $0.5 \mathrm{~s}$. The examination lasted only $5.6 \mathrm{~s}$.

The CT perfusion source images were sent to a dedicated satellite workstation (Advantage Workstation 4.6 software, CT Perfusion 4D, GE Health Care, Chicago, Illinois, USA) for post-processing, where the plane of symmetry was manually set; CBF, CBV, MTT, and TTP maps were generated, and, based on the threshold parameters, the system identified the areas of reversible and irreversible damage in a semi-automated manner.

For each patient, CTA-SI ASPECTS were calculated retrospectively. Using local PACS workstations (Impax Client, Agfa Heathcare NV, Mortsel, Belgium), transverse images from the angio-CT were reconstructed in layers $0.625 \mathrm{~mm}$ thick, using a soft-tissue algorithm with the window that best reflected the contrast enhancement of pial and arachnoid vessels, perforators, and medullary veins.

The status and presence of collaterals were also evaluated retrospectively, using images of CT angiography reconstructed with the same workstations, employing projections of maximum intensity in the axial and frontal plane in order to best predict asymmetry.

The evaluation of haemorrhagic complications was based on ECASS I criteria. To classify them, $\mathrm{PH} 1$ and $\mathrm{PH} 2$ classes were adopted, dividing the patients into two groups: with or without parenchymal haematoma. During the procedures, total radiation doses and fluoroscopy times were recorded along with the amount and concentration of the relevant contrast agent. The clinical outcome of the treatment was based on a modified Rankin scale, with a result after 90 days above 2 regarded as unfavourable and below or equal to 2 as good.

\section{Statistical analysis}

The obtained results were presented as means and standard deviations in the case of quantitative variables with a normal distribution and as medians and interquartile values for ordinal and quantitative variables with different distributions. Nominal variables were presented as percentages. Using the Mann-Whitney $U$ test and Spearman's correlation, the relationship between clinical results and SMCTS in the group representing successful recanalisation was verified. Using the same method, the relationship between SMCTS, radiologic outcomes, and the risk of haemorrhagic complications was assessed. Differences in the radiation exposure of patients and the burden of contrast agents were analysed using ANOVA and the Kruskal-Wallis test. A result of $p<0.05$ was considered statistically significant for all tests. Statistical analysis of the results was carried out using Dell Statistica version 13 (Dell, Round Rock, Texas, USA).

\section{Results}

During the study period, 50 patients were disqualified from further analysis, mainly $(n=42)$ due to partially unavailable imaging data (in 29 patients, perfusion CT was lacking; in 2 patients the examination was non-diagnostic; 1 patient lacked CT angiography and for 10 patients, both perfusion and angio CT scans were unavailable). Eight patients suffered from stroke in the vertebrobasilar system; 1 patient was excluded from the study due to bilateral closure of large anterior circulation vessels. Data on radiation doses and contrast agents were unavailable for 10 patients; however, since treatment exposure was a secondary goal, these patients were excluded from the burden analyses only. Following verification of the availability and quality of imaging examinations, 85 patients were included in the study. The characteristics of these patients are presented in Table I.

In 50 (59\%) patients, 90 days after the onset of symptoms, the modified Rankin scale score was below or equal to 2. Of patients not in this group, 18 died, 16 of them during hospitalisation. Patients with good long-term outcomes differed from those with unsatisfactory results of treatment in that the former were younger and also achieved lower scores on the NIHSS scale. Differences in gender were noted in the study group, along with time from the onset of symptoms to the beginning of treatment, frequency of atrial fibrillation, and the presence of ischaemic heart disease or diabetes; however, these differences were without statistical significance.

The incidence of hypertension, the percentage of thrombolysis, and BMI were similar in both groups. In the group with good long-term results, revascular- 
isation procedures were more frequently successful (Table II).

Forty-nine $(49$, or $57 \%)$ patients achieved SMCTS scores of 0; the scores of $16(19 \%)$ patients equalled 1. Only 7 (8\%) patients scored all three points; however, none of them obtained a favourable modified Rankin scale score within the 3-month observation period. In the remaining groups, the rate of good clinical outcomes ranged between 61.5 and $65.3 \%$; the difference between patients with SMCTS $=3$ and groups with lower results was statistically significant ( $p=0.00362)$, but the difference between individual subpopulations was not.

Figure 1 presents long-term treatment results in relation to SMCTS values.

In the group with the highest SMCTS scores, we observed frequent occurrence $(28.6 \%)$ of haemorrhagic complications, compared to $10.3 \%$ in the remaining patients, and a significantly lower level of radiological effectiveness of procedures $(43 \%$ compared to $67 \%$ ). Calculated odds ratios were 3.5 and 0.38 , respectively; however, these ratios were without statistical significance, as reflected by $p$ values of 0.16 and 0.21 .

The clinical outcome was dependent on the result of thrombectomy. This association was observed for the entire group ( $p$-value for the $U$ test $=$ 0.00075 , correlation rho $=0.43, \mathrm{OR}=6.83$ ) and for all subpopulations except patients with SMCTS of 3 . The incidence of haemorrhage was not correlated in the observed group with SMCTS, administration of fibrinolytic agent, effectiveness of recanalisation, or parameters of the procedure such as fluoroscopy time, radiation dose, or contrast agent dose.

Table I. Clinical characteristics of the study group $(n=85)$

\begin{tabular}{|lc|}
\hline Parameter & Result \\
\hline Age & $69(58-77)$ \\
\hline Sex (female) & $39(46 \%)$ \\
\hline Occlusion site: & $52(61 \%)$ \\
\hline M1 & $18(21 \%)$ \\
\hline M2 & $4(5 \%)$ \\
\hline ICA & $11(13 \%)$ \\
\hline Diandem & $24(28 \%)$ \\
\hline Atrial fibrillation & $37(44 \%)$ \\
\hline Hypertension & $66(78 \%)$ \\
\hline Ischaemic heart disease & $30(35 \%)$ \\
\hline NIHSS at admission & $16(12-19)$ \\
\hline Intravenous thrombolysis & $66(78 \%)$ \\
\hline
\end{tabular}

M1-M1 segment of the middle cerebral artery from its origin to bifurcation/ trifurcation, M2 - M2 segment of the middle cerebral artery, also known as the insular segment, ICA - internal carotid artery, NIHSS - National Institute of Health Stroke Scale; clinical measure of stroke/neurologic deficit; the scale includes values in the range 0-42; an NIHSS score > 21 is assumed to represent a severe stroke, TANDEM - internal carotid artery and middle cerebral artery occlusion in the entire segment with or without visible blood flow in between (through the ophthalmic artery or either communicating artery).

Table II. Group comparison of good vs. poor clinical outcomes

\begin{tabular}{|lccc|}
\hline Variable & $\begin{array}{c}\text { Good clinical outcome } \\
\text { MRS } \leq 2, n=50\end{array}$ & $\begin{array}{c}\text { Poor clinical outcome } \\
\text { MRS }>2, n=35\end{array}$ & $P$-value \\
\hline NIHSS, median (IQR) & $14.5(11-18)$ & $17.5(14-19)$ & 0.029 \\
\hline Age, median (IQR) & $67(51.75-73.5)$ & $72(66.5-80)$ & 0.004 \\
\hline Recanalisation (TICl 2b or 3; $n(\%))$ & $41(82 \%)$ & $14(40 \%)$ & 0.004 \\
\hline Time to treatment, median (IQR) [min] & $209(165.5-260)$ & $238(172.25-297)$ & 0.228 \\
\hline Ischaemic heart disease, $n(\%)$ & $15(30 \%)$ & $15(42.86 \%)$ & 0.317 \\
\hline Myocardial infarction, $n(\%)$ & $4(8 \%)$ & $7(20 \%)$ & 0.353 \\
\hline Sex (female), $n(\%)$ & $21(42 \%)$ & $18(51.43 \%)$ & 0.463 \\
\hline Atrial fibrillation, $n(\%)$ & $20(40 \%)$ & $17(48.57 \%)$ & 0.508 \\
\hline Diabetes, $n$ (\%) & $13(26 \%)$ & $11(32.43 \%)$ & 0.673 \\
\hline BMI, median (IQR) & $27.68(24.78-31.22)$ & $28.68(25.48-30.12)$ & 0.699 \\
\hline Hypertension, $n(\%)$ & $38(76 \%)$ & $28(80 \%)$ & 0.759 \\
\hline Intravenous thrombolysis, $n(\%)$ & $39(78 \%)$ & $27(77.14 \%)$ & 0.947 \\
\hline
\end{tabular}




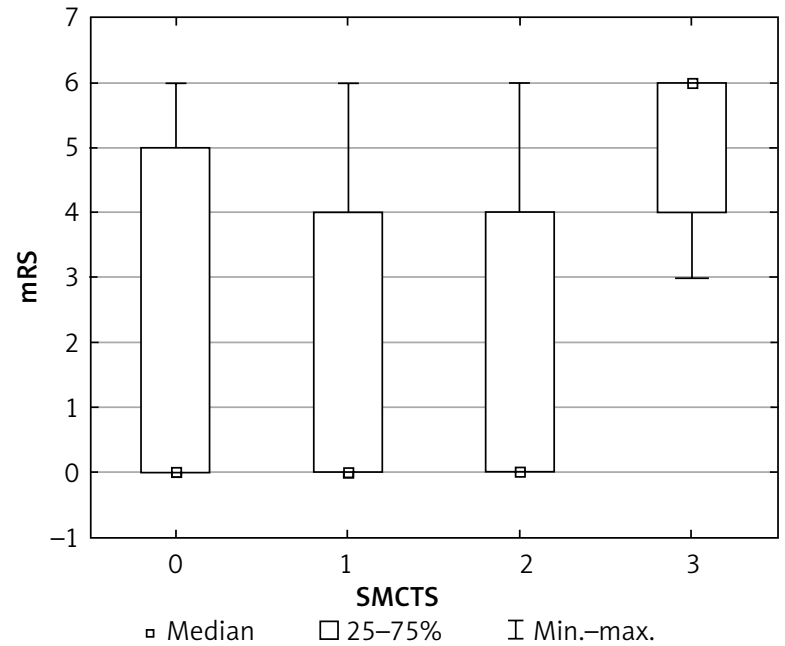

Figure 1. Proportion of good and poor clinical outcomes in SMCTS groups. Box and whiskers chart in SMCTS groups

In the study population, particular attention was paid to the subgroup of 29 patients with intermediate values of SMCTS, where strong negative dependence was observed between the effectiveness of reperfusion and the risk of haemorrhagic transformation $(p=0.008$, rho $=0.506)$, despite the similar general incidence of this complication in patients in the remaining groups. In this group, a good mRS result within the 3-month observation period was observed in $60 \%$ of cases. Haemorrhagic stroke transformation in this group occurred only in patients with unsuccessful thrombectomies; this was not associated with either lack of collateral circulation or burden of endovascular surgery. Nor did the two latter factors influence frequency of bleeding in the whole study population. Among patients in the intermediate SMCTS subgroup with and without haemorrhage, there were no differences in patients' ages, baseline NIHSS, co-morbidities, the rate of thrombolysis, or time until treatment (Table III).

\section{Discussion}

The most important finding of our study is the high predictive value of SMCTS for poor clinical outcomes. This is very useful information for patients who are in doubt about the indications for intervention, because a lack of potential benefits, in connection with the burden of therapy, has a definite influence on the course of treatment. Older patients, often with numerous comorbidities, are those most frequently treated, and information of this kind at the beginning of the therapeutic process can be crucial.

The statistically insignificant correlation of lower SMCTS values with the clinical outcome of the treatment should be attributed primarily to the simplified evaluation of perfusion maps described above, which randomly inflates the CBV-ASPECTS values. Also disappointing is the lack of any relationship between the analysed radiological indicators and the risk of haemorrhage, the effectiveness of reperfusion, or the burden associated with thrombectomy.

In parallel with studies on the reduction of the time from the first symptoms to the start of treatment, studies were conducted on the optimisation of qualifications for reperfusion, so as not to miss any potentially reversible ischaemia while avoiding unnecessary exposure of patients who can no longer benefit from treatment. The analysis of CTA-SI images, despite the interest it aroused at the beginning

Table III. Haemorrhages in SMCTS 1 and 2 patients

\begin{tabular}{|lccc|}
\hline Factor & $\begin{array}{c}\text { Insignificant bleeding } \\
\text { or none }\end{array}$ & $\begin{array}{c}\text { Clinically significant } \\
\text { haemorrhage }\end{array}$ & $P$-value \\
\hline Reperfusion, $n(\%)$ & $20(77 \%)$ & 0 & 0.008 \\
\hline Atrial fibrillation, $n(\%)$ & $7(27 \%)$ & $1(33 \%)$ & 0.853 \\
\hline Age, median (IQR) & $59(50-78)$ & $60(59-66)$ & 0.693 \\
\hline NIHSS, median (IQR) & $15(13-20)$ & $18(15-19)$ & 0.857 \\
\hline Intravenous thrombolysis, $n(\%)$ & $22(85 \%)$ & $2(67 \%)$ & 0.477 \\
\hline Collaterals, $n(\%)$ & $15(58 \%)$ & $240(233-278)$ & 0.801 \\
\hline Time to treatment, median (IQR) & $224(176-299)$ & 0 & 0.333 \\
\hline Sex (female), $n(\%)$ & $11(42 \%)$ & 0.173 \\
\hline
\end{tabular}


of the century, is rarely used today. Although its efficacy in assessing the extent of irreversible ischaemia, its ability to predict the final infarction territo$r y$, and its convergence with the diffusion-weighted imaging picture in acute and hyperacute ischaemic stroke have all been confirmed, the number of publications related to this technique is decreasing, presumably due to the development of more functional techniques such as CT and MR perfusion.

The advantage of perfusion in both $C T$ and $M R$ is the well-established relationship between blood flow measurements and the pathophysiology of cerebral ischaemia. The terms core of ischaemia, penumbra, and oligaemia are directly related to specific perfusion disorders, which can be measured in a series of scans following a standardised injection of the contrast agent, mathematically modelled, and mapped [9-12].

Nevertheless, a perfusion examination does not categorically dictate a change of treatment, nor does it enable an unequivocal prognostic assessment. Most studies on the mechanical treatment of ischaemic strokes are conducted without evaluating brain perfusion. Some of the indicators marked in perfusion depend on the mathematical algorithms used in the software and vary among manufacturers [13]. Another disadvantage of the perfusion examination involves temporal requirements, since currently its use, data processing, and interpretation take more time than acquisition $\mathrm{CT}$ and angioCT scans.

In the present study, SMCTS values were calculated retrospectively by the authors and then evaluated in the context of clinical results of the treatment. In practice, however, the routine use of SMCTS can be a problem, as it involves perfusion examinations, which are not part of the standard protocol in most centres [13], and calculation of scores on three other scales with which the on-duty radiologist may not have experience. Moreover, specific changes in management only affect patients with the highest SMCTS, i.e. about $8 \%$ of patients, which further limits the usefulness of this measure.

The number of patients with intermediate SMCTS exceeded $1 / 3$ of the study group. We cannot fail to address the protective effects of successful thrombectomy, such as a lower risk of haemorrhage transformation. This finding is inconsistent with the majority of theories regarding the pathomechanism of haemorrhage, associating this complication with reperfusion injury $[14,15]$. A significantly lower rate of atrial fibrillation in this group may suggest that strokes with a non-cardiac aetiology may be associated with a lower risk of haemorrhage. It cannot be ruled out that patients with arrhythmias had been treated previously with vitamin $\mathrm{K}$ antagonists a practice not evaluated in our study - and that their effects are associated with greater susceptibility to haemorrhage. It should be mentioned, however, that in the case of anticoagulation, the effectiveness of vitamin $\mathrm{K}$ antagonists is usually monitored using biochemical tests, such as the so-called therapeutic INR, in the range 2-3. Such patients would be disqualified from thrombolysis, while no differences in the percentages of patients after fibrinolysis between groups with or without haemorrhage were observed. Most likely, the negative correlation between reperfusion and haemorrhage is also connected with the ECASS I scale used in our study and the adoption of only the highest grades for consideration as haemorrhage. One question that remains unanswered is why thrombectomy should change the prognosis in this specific setup.

Our study is limited, as it was retrospective and based on a single centre, and as only a group of patients treated by mechanical thrombectomy was analysed. The number of patients also suggests that the results should be interpreted with caution. Only 10 patients experienced haemorrhagic transformation of stroke, and even fewer patients were characterised by the highest SMCTS. For comparison, in the initial records of the score, 150 patients experienced a good outcome, and 24 (16\%) patients were rated at $\mathrm{CMCTS}=3$.

\section{Conclusions}

Although the principle of 'time is brain' is still valid in the treatment of acute stroke, the optimisation of the therapeutic process requires advanced neuroimaging. SMCTS turns out to be an accurate predictor of therapy failure, mainly in the long (mRS) but also in the short term (recanalisation rate, haemorrhagic transformation). We believe that multimodal CT scoring, even in a limited version that can be obtained with a 4-cm perfusion field (SMCTS), is useful in the qualification of patients at the limit of indications.

\section{Conflict of interest}

The authors declare no conflict of interest. 


\section{References}

1. Palaniswami M, Yan B. Mechanical thrombectomy is now the gold standard for acute ischemic stroke: implications for routine clinical practice. Interv Neurol 2015; 4: 18-29.

2. Berkheimer OA, Fransen PSS, Beumer D, et al. A randomized trial of intraarterial treatment for acute ischemic stroke. $\mathrm{N}$ Engl J Med 2015; 372: 11-20.

3. Campbell BCV, Mitchell PJ, Kleinig TJ, et al. Endovascular therapy for ischemic stroke with perfusion-imaging selection. N Engl J Med 2015; 372: 1009-18.

4. Goyal M, Demchuk AM, Menon BK, et al. Randomized assesment of rapid endovascular treatment of ischemic stroke. N Eng J Med 2015; 372: 1019-30.

5. Saver JL, Goyal M, Bonafe A, et al. Stent-retriever thrombectomy after intravenous t-PA vs. t-PA alone in stroke. N Eng J Med 2015; 372: 2285-95.

6. Campbell BCV, Donnan G, Mitchell PJ, et al. Endovascular thrombectomy for stroke: current best practice and future goals. Stroke Vasc Neurol 2016; 1: 16-22.

7. Powers WJ, Derdeyn CP, Biller J, et al. 2015 American Heart Association/American Stroke Association focused update of the 2013 guidelines for the early management of patients with acute ischemic stroke regarding endovascular treatment: a guideline for healthcare professionals from the American Heart Association/American Stroke Association. Stroke 2015; 46: 3020-35.

8. de Rueda ME, Parilla G, Manzano-Fernandez S, et al. Combined multimodal computed tomography score correlates with futile recanalization after thrombectomy in patients with acute stroke. Stroke 2015; 46: 2512-29.

9. Schramm P, Schellinger P D, Fiebach JB, et al. Comparison of $\mathrm{CT}$ and $\mathrm{CT}$ angiography source images with diffusion-weighted imaging in patients with acute stroke within 6 hours after onset. Stroke 2002; 33: 2426-32.

10. Coutts SB, Lev MH, Elasziw M, et al. ASPECTS on CTA source images versus unenhanced $C T$ added value in predicting final infarct extent and clinical outcome. Stroke 2004; 35: 2472-6.

11. Wintermark M, Flanders AE, Velthuis B, et al. Perfusion-CT assesment of infarct core and penumbra: receiver operating characteristic curve analysis in 130 patients suspected of acute hemispheric stroke. Stroke 2006; 37: 979-85.

12. von Kummer R, Dzialowski I. Imaging of cerebral ischemic edema and neuronal death. Neuroradiology 2017; 59: 545-53.

13. Heit JJ, Wintermark M. Perfusion computed tomography for the evaluation of acute ischemic stroke strengths and pitfalls. Stroke 2016; 47: 1153-8.

14. Berger C, Fiorelli M, Steiner T, et al. Hemorrhagic transformation of ischemic brain tissue. Stroke 2001; 32: 1330-5.

15. Bai J, Lyden PD. Revisiting cerebral postischemic reperfusion injury: new insights in understanding reperfusion failure, hemorrhage, and edema. Int J Stroke 2015; 10: 143-52.

Received: 3.01.2019, accepted: 10.03.2019. 